\title{
NT5E Genetic Mutation Is a Rare But Important Cause of Intermittent Claudication and Chronic Limb-Threatening Ischemia
}

\author{
Nobuyoshi Azuma, MD, PhD; Tetsuro Uchida, MD, PhD; Shinsuke Kikuchi, MD, PhD; \\ Mitsuaki Sadahiro, MD, PhD; Tsunehiro Shintani, MD; Kumiko Yanagi, PhD; \\ Ryuji Higashita, MD, PhD; Atsushi Yamashita, MD; \\ Yoshio Makita, MD, PhD; Tadashi Kaname, MD, PhD
}

Background: NT5E genetic mutations are known to result in calcification of joints and arteries (CALJA), and worldwide, 14 patients from 7 families have been reported.

Methods and Results: A total of 5 patients from 2 independent families with CALJA were found in Japan. Of them, 3 complained of intermittent claudication (IC), and 1 suffered from bilateral chronic limb-threatening ischemia (CLTI). Whole-exome sequencing analysis revealed an identical mutation pattern (c.G3C on the exon 1 start codon) that was unique compared with NT5E mutations reported in other countries.

Conclusions: Vascular specialists need to recognize CALJA as a rare cause of ischemic IC and CLTI.

Key Words: Calcification of joints and arteries; Chronic limb-threatening ischemia; Intermittent claudication; NT5E gene; Peripheral artery calcification

$\mathbf{H}$ eavy calcification of aneurysmally dilated arteries combined with joint calcification, so-called calcification of joints and arteries (CALJA), was first described in 1912 by Magnus-Lavy, ${ }^{1}$ and 7 cases were reported before 2011,25 however, the molecular and genetic background of CALJA was unknown until St. Hilaire's report in $2011 .{ }^{6}$ Since they unveiled mutations of the NT5E ( $5^{\prime}$-ectonucleotidase) gene as a cause of CALJA, ${ }^{6} 14$ patients from 7 families have been reported around worldwide (Table). ${ }^{7-10}$ Heavily calcified occluded arteries of the lower extremities caused intermittent claudication (IC) as an ischemic symptom in many of these patients, but there is no literature on CALJA patients needing vascular reconstruction for limb salvage. Herein, we report 2 families with identical genetic mutation patterns, and 3 patients needed distal bypass surgeries, including 1 patient with chronic limb-threatening ischemia (CLTI) who had already lost her lower extremity before being diagnosed with CALJA.

This report provides information on rare genetic disorders of arterial calcification caused by a deficiency of CD73 (ACDC) and addresses key issues that will contribute to a better understanding of the pathogenesis of arterial calcification and aneurysmal dilatation, ${ }^{11}$ both of which are still unsolved but very important vascular pathologies.

\section{Methods}

To clinically diagnose CALJA, we performed the following examinations to indicate calcification of the arteries and joints: X-ray imaging of the hands and lower extremities, and computed tomography (CT) scans. To evaluate ischemia of the lower extremities, measurement of the anklebrachial pressure index (ABI) or skin perfusion pressure of the foot was performed.

For genetic analysis, genomic DNA was extracted from whole blood cells using a QIAamp DNA Blood Mini Kit (QIAGEN, Hilden, Germany). We performed whole-exome sequencing analysis as previously described..$^{12}$ In brief, we used the SureSelect Human All Exon V6 kit (Agilent Technology, Santa Clara, CA, USA) for capture and a HiSeq2500 (Illumina, San Diego, CA, USA) for sequencing. Reads were aligned to GRC37 using Burrows-Wheeler

Received February 26, 2020; revised manuscript received April 29, 2020; accepted May 21, 2020; J-STAGE Advance Publication released online June 11, 2020 Time for primary review: 14 days

Department of Vascular Surgery (N.A., S.K.), Education Center (Y.M.), Asahikawa Medical University, Asahikawa; Division of Cardiovascular Surgery, Department of Surgery II, Yamagata University, Yamagata (T.U., M.S., A.Y.); Department of Vascular Surgery, Shizuoka Red Cross Hospital, Shizuoka (T.S.); Department of Genome Medicine, National Center for Child Health and Development, Tokyo (K.Y., T.K.); and Department of Cardiovascular Surgery, Yokohama General Hospital, Yokohama (R.H.), Japan

Mailing address: Nobuyoshi Azuma, MD, PhD, Department of Vascular Surgery, Asahikawa Medical University, 2-1 Midorigaokahigashi, Asahikawa 078-8510, Japan. E-mail: nazuma@asahikawa-med.ac.jp

ISSN-1346-9843 All rights are reserved to the Japanese Circulation Society. For permissions, please e-mail: cj@j-circ.or.jp 


\begin{tabular}{|c|c|c|c|c|c|c|c|c|}
\hline \multirow{2}{*}{$\begin{array}{c}\text { Family } \\
\text { no. }\end{array}$} & \multicolumn{2}{|c|}{ Patients } & \multirow{2}{*}{$\begin{array}{l}\text { Lwoer limb } \\
\text { sysmptoms }\end{array}$} & \multirow{2}{*}{$\begin{array}{c}\text { Nationality } \\
\text { (region) }\end{array}$} & \multicolumn{3}{|c|}{ Mutation of NT5E (NM_002526) } & \multirow{2}{*}{$\begin{array}{l}\text { Ref } \\
\text { no. }\end{array}$} \\
\hline & Age & Sex & & & Exon & $\begin{array}{c}\text { Variant } \\
\text { (zygocity) }\end{array}$ & $\begin{array}{c}\text { Predicted AA } \\
\text { change }\end{array}$ & \\
\hline \multirow[t]{5}{*}{1} & $54^{\#}$ & $\mathrm{~F}$ & Rest pain & USA & 3 & c. $662 \mathrm{C}>\mathrm{A}$ (homo) & p.Ser221Ter & 6 \\
\hline & 53 & M & IC & & & & & \\
\hline & 51 & M & IC & & & & & \\
\hline & 49 & $\mathrm{~F}$ & IC & & & & & \\
\hline & 44 & $\mathrm{~F}$ & IC & & & & & \\
\hline \multirow[t]{3}{*}{2} & $68^{\#}$ & $\mathrm{~F}$ & Leg pain & USA & 5 & c.1073G>A (homo) & p.Cys358Tyr & 6 \\
\hline & 73 & $\mathrm{~F}$ & Leg pain & & & & & \\
\hline & 70 & $\mathrm{~F}$ & Leg pain & & & & & \\
\hline \multirow[t]{2}{*}{3} & $44^{\#}$ & $\mathrm{~F}$ & IC & USA & 9 & c.1609dupA & p.Val537SerfsTer7 & 6 \\
\hline & & & & & 3 & c. $662 \mathrm{C}>\mathrm{A}$ (hetero) & p.Ser221Ter & \\
\hline \multirow[t]{2}{*}{4} & $54^{\#}$ & $\mathrm{~F}$ & IC & Japan (Tokyo) & 1 & c. $3 \mathrm{G}>\mathrm{C}$ (hetero) & p.Met1? & 7 \\
\hline & & & & & 2 & c. $373 \mathrm{G}>\mathrm{C}$ (hetero) & p.Glu125Ter & \\
\hline \multirow[t]{2}{*}{5} & $26^{\#}$ & M & None & China & 7 & c. $1360 \mathrm{G}>\mathrm{A}$ & p.Gly454Arg & 8 \\
\hline & 30 & $\mathrm{~F}$ & None & & 8 & c. $1387 \mathrm{C}>\mathrm{T}$ (hetero) & p.Arg463Ter & \\
\hline 6 & $42^{\#}$ & M & IC & Netherlands & Intron 3 & c. $751+2 \mathrm{~T}>\mathrm{C}$ (homo) & ND & 9 \\
\hline 7 & $66^{\#}$ & M & IC & Japan (Chiba) & 1 & $\begin{array}{c}\text { c.3G>C (homo) } \\
\text { speculated }\end{array}$ & p.Met1? & 10 \\
\hline \multirow[t]{3}{*}{8} & $70^{\#}$ & $\mathrm{~F}$ & CLTI & Japan (Shizuoka) & 1 & c. $3 G>C$ (homo) & p.Met1? & \\
\hline & 80 & M & None & & & & & \\
\hline & 74 & $\mathrm{~F}$ & IC & & & & & \\
\hline \multirow[t]{2}{*}{9} & $81^{\#}$ & $\mathrm{~F}$ & IC & Japan (Yamagata) & 1 & c. $3 G>C$ (homo) & p.Met1? & \\
\hline & 74 & $\mathrm{~F}$ & IC & & & & & \\
\hline
\end{tabular}

"Proband; AA, amino acid; CLTI, chronic limb-threatening ischemia; IC, intermittent claudication; ND, not described; Ref, reference.

Aligner (http://bio-bwa.sourceforge.net/). Variants were named using the GATK Unified Genotyper and using ANNOVA (http://annover.openbioinfomatics.org/en/latest/).

This study was approved by the ethics committees of the Asahikawa Medical University and Yamagata University and followed the Declaration of Helsinki and the ethical standards of the responsible committee on human experimentation. The genetic analysis of this study was performed as one of the Initiative of Rare and Undiagnosed Diseases (IRUD) projects. All patients and their family members signed informed consent documents and agreed to clinical and genetic examinations, and they also agreed to publish their case details and images.

\section{Results}

In this IRUD project, we found 2 independent families with clinically diagnosed CALJA arising from NT5E mutations.

\section{Family 1}

Family 1 (Family no. 8 in Table) included 3 patients diagnosed with CALJA (Figure 1A). The proband, Patient II-10 was a 70 -year-old woman with a 20 -year history of IC. Her right lower leg was amputated below the knee in a previous hospital due to infection of ischemic ulcers of the forefoot. During the postoperative term, ulcers appeared on multiple toes of the left foot (Figure 1B). Her ABI was unmeasurable with no pulse wave and skin perfusion pressure was $16 \mathrm{mmHg}$ on the dorsum and $9 \mathrm{mmHg}$ on the plantar surface. Plain radiography demonstrated heavy calcification with areas of arteriomegaly in the lower extremity (Figure 1C-E) and a partially calcified artery around the elbow and hand joints. Metacarpal phalangeal and interphalangeal joint calcification was also found (Figure 1F,G). CT and digital subtraction angiography demonstrated extensive arterial occlusions between the external iliac artery and popliteal artery, as well as the malleolar arteries (Figure 1H). Left common iliac arteryposterior tibial artery (PTA) bypass using a great saphenous vein (GSV) was performed (Figure 1H). Although minor amputations (her 1st and 4th toe) were needed, complete ulcer healing was achieved (Figure 1B). She regained her ability to walk with a prosthesis and has continued walking for 2 years after bypass surgery. Because the findings from the series of examinations were clearly distinguished from those usually observed with peripheral artery disease (PAD), we investigated the X-ray screening and measuring the ABIs of her family members: 2 siblings of the proband had arteriomegaly with severe calcification in their lower extremities (Figure 1I,J). Patient II-1 was asymptomatic and had an ABI of 0.75 . Patient II- 8 had IC with an ABI of 0.6. Neither patient had atherosclerotic risk factors and so they received conservative treatment. They were finally diagnosed with CALJA based on NT5E mutation by genetic testing.

\section{Family 2}

Family 2 (Family no. 9 in Table) had sisters with CALJA aged 81 and 74 years, respectively (Figure 2A). The 81-year-old woman presented with a 50-year history of joint pain in her legs and a history of left femoro-PTA bypass surgery performed 5 years ago for IC. Although she regularly visited an outpatient clinic for moderate aortic 
A

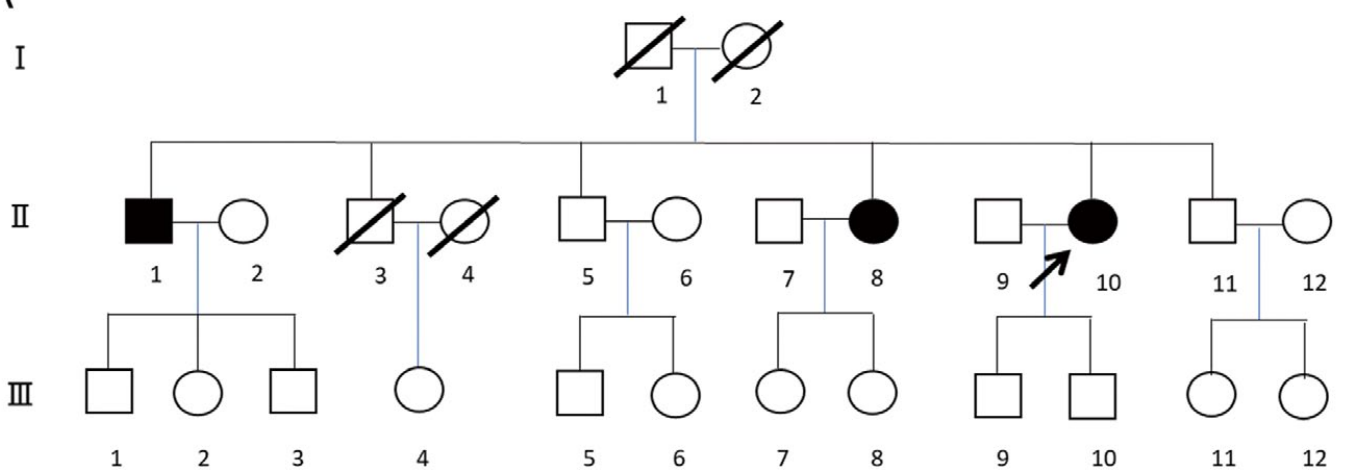

Patient II.10

Patient II.1

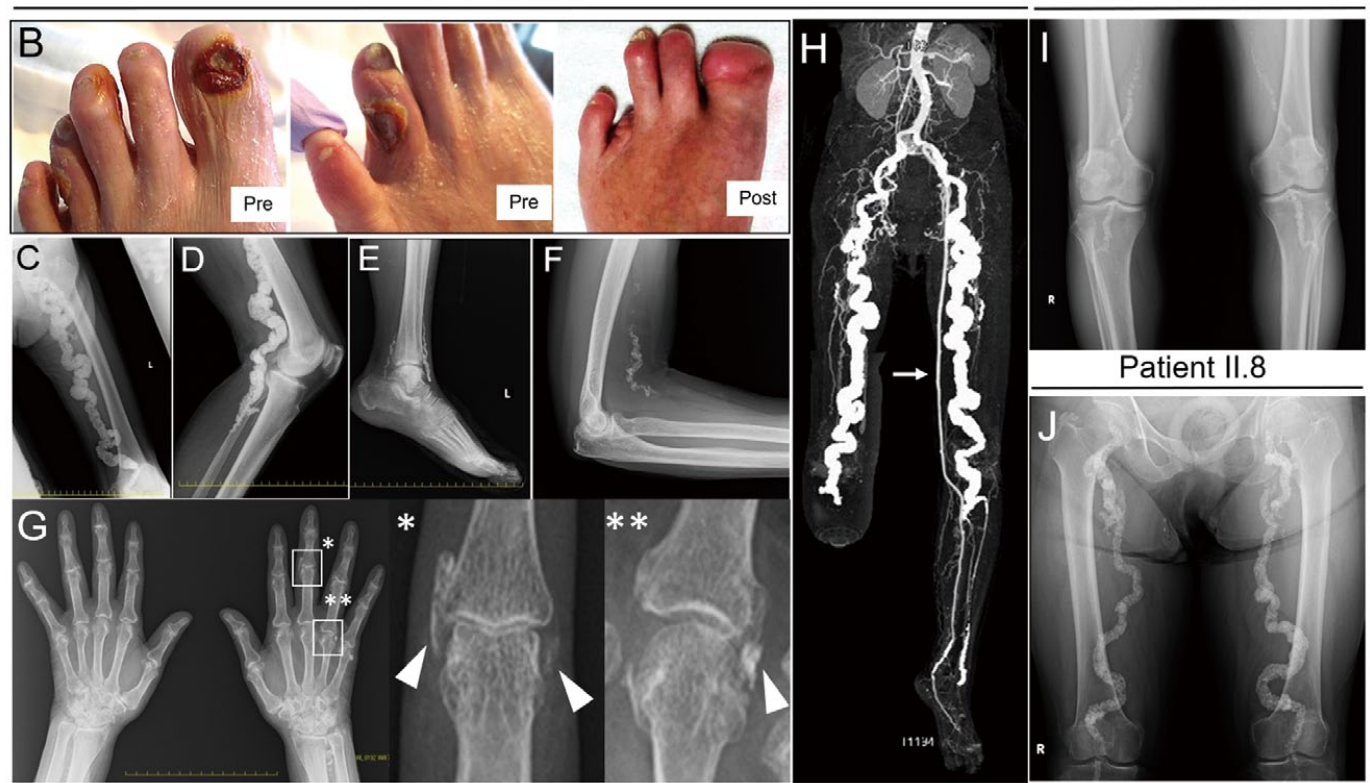

Figure 1. Pedigree and the clinical and radiographic findings in Family 1. (A) Pedigree. Open symbols indicate unaffected family members, and solid symbols are affected members. Arrows indicate the probands. Squares indicate male family members, circles female members, and slashed symbols are for deceased members. (B) Pre- and postoperative appearance of the left forefoot of Patient II-10. (C-F) Heavily calcified left femoral, popliteal, paramalleolar, and brachial arteries on plain radiographs of Patient II-10. (G) Interphalangeal $\left({ }^{*}\right)$ and metacarpal $\left(^{* *}\right)$ joint calcification. $(\mathbf{H})$ CT angiography image of the same patient after common iliac-posterior tibial artery bypass surgery. White arrow indicates the bypass graft. Bilateral lower limb arteries from the external iliac to popliteal arteries are aneurysmally dilated and occluded with extensive heavy calcification. Right lower limb was amputated previously. Heavily calcified arteries of the lower extremity arteries are also observed on plain radiographs of Patient II-1 (I) and Patient II-8 (J) in the same family.

valve stenosis (AS), her exertional fatigue gradually worsened. Echocardiography revealed severe AS, so aortic valve replacement (AVR) was performed. Intraoperatively, both the aortic valve leaflets and the valvular annulus showed significant calcification. The IC of her right leg worsened 3 years after AVR. The ABI value showed no wave (unmeasurable) and plain radiography of the lower extremities revealed significant calcification with areas of arteriomegaly (Figure 2B). Femoro-tibioperoneal trunk bypass surgery was performed using a GSV. CT angiography revealed extensive occlusion of the bilateral femoropopliteal arteries accompanied by aneurysmal changes with severe calcification and patent saphenous vein grafts (Figure 2C).
Metacarpal phalangeal joint calcification was also found (Figure 2D). The 74-year-old woman (Patient II-11) reported a 10-year history of bilateral IC in her calves. At 71 years of age, she was diagnosed with severe AS, and AVR was performed. During the follow-up, she continued to have IC. The ABI value was 0.39 for the right leg and 0.32 for the left leg. Plain radiography (Figure 2E) and CT revealed similar findings to those observed in her sister, such as occluded aneurysmal femoropopliteal arteries with dense calcification (Figure 2F), and she underwent bilateral femoro-tibial artery bypass surgeries for IC. Currently, both patients are doing well. Both patients were diagnosed with CALJA based on NT5E mutation by genetic testing. 


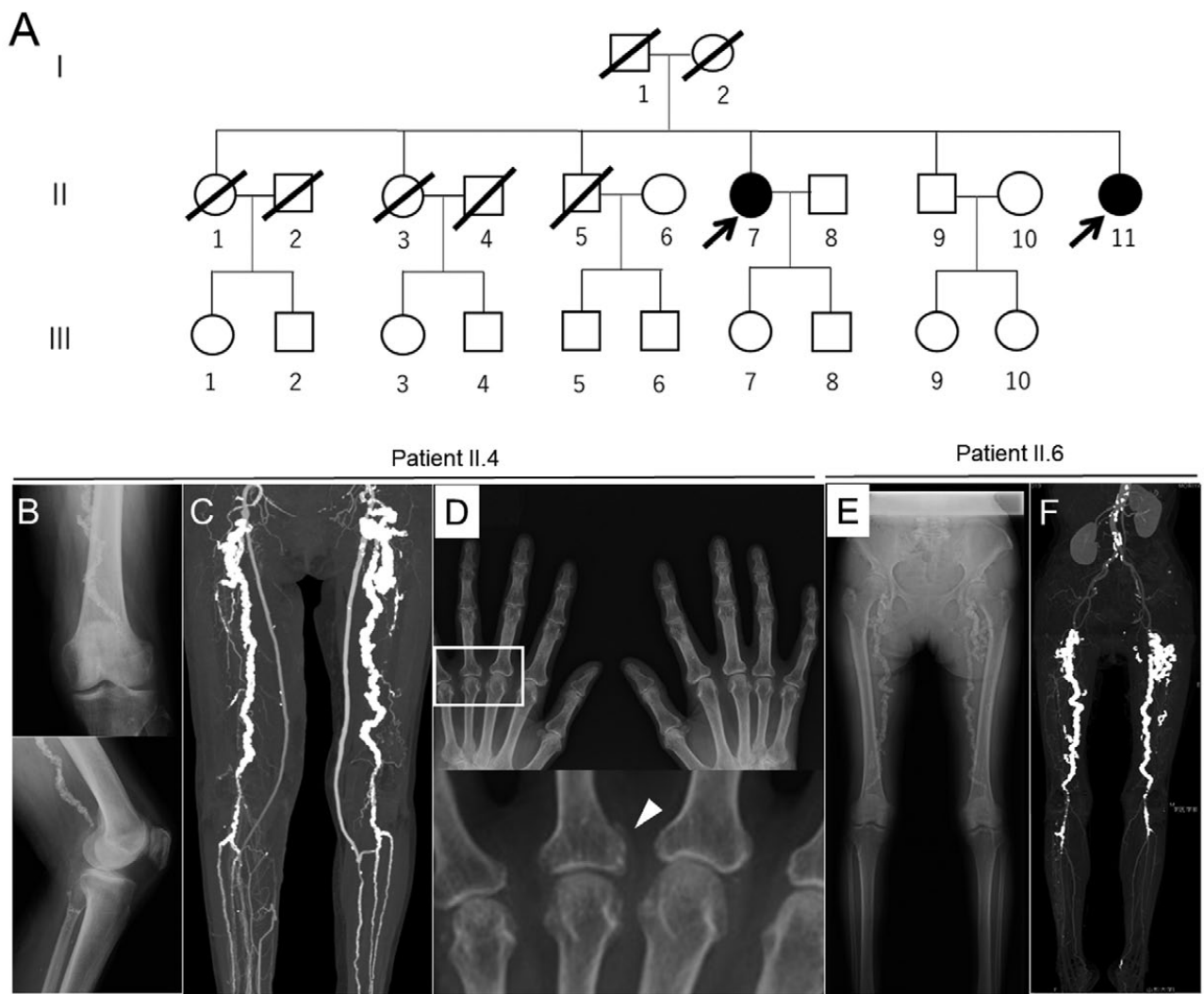

Figure 2. Pedigree and the clinical and radiographic findings in Family 2. (A) Pedigree. Open symbols indicate unaffected family members, and solid symbols are affected members. Arrows indicate the probands. Squares indicate male family members, circles female members, and slashed symbols are for deceased members. (B) Heavily calcified femoral artery on plain radiographs of Patient II-4. (C) CT angiography image of the same patient after bypass surgeries of both lower limbs. Bilateral femoral arteries are aneurysmally dilated and occluded with extensive heavy calcification. (D) Metacarpal joint calcification (arrowhead). Patient II-6 also has extensive calcification in the bilateral superficial and deep femoral arteries shown on plain radiography (E) and CT angiography (F).

\section{Genetic Analysis}

After filtering the variants between patients and nonpatients, we identified a homozygous variant of NT5E, NM_002526: c.3G>C (p.Met1?), in the patients (Figure 3). The homozygous variant was not found in the Japanese genome variant database (HGVD; http://www.hgvd. genome.med.kyoto-u.ac.jp) or in-house data of wholeexome sequencing. The variant was confirmed by Sanger sequencing. The homozygous variant and patients were cosegregated in these families (unpublished data).

\section{Discussion}

We found a homozygous variant of c. $3 \mathrm{G}>\mathrm{C}$ in NT5E in 2 families with CALJA. According to the Genome Aggregation Database (gnomAD: https://gnomad.broadinstitute. org/), the allele frequency of the variant in the East Asian population is $1 / 1,560$. Thus, the prevalence of affected patients with the homozygous variant is estimated to be approximately 1/2,500,000 in the East Asian population.

The NT5E gene encodes the cell surface protein CD73, which enzyme catalyzes conversion of adenosine monophosphate (AMP) to adenosine. Dysfunction of CD73 leads to reduced adenosine production, which results in vascular calcification through tissue-nonspecific alkaline phosphatase (TNAP). ${ }^{13}$ Jin et al proved that increased activity of TNAP compensated for reduced adenosine production in an experimental disease model using CALJA patient-specific induced pluripotent stem cell technology. ${ }^{11}$ TNAP is known to hydrolyze inorganic pyrophosphate (PPi), a calcification inhibitor, and lower cellular PPi concentrations to promote calcification. Although the molecular mechanism of aneurysmal dilatation due to $N T 5 E$ deficiency is not well understood, Markello et al reported their histological findings of arterial specimens that showed calcification and disruption of the internal elastic lamina, with clusters of osteoclast-like cells. ${ }^{14}$ Endothelial CD73 plays an important role in the prevention of inflammation, atherosclerosis and calcification of vascular tissues. ${ }^{15}$ This evidence suggests that CD73 dysfunction results in arterial calcification, aneurysmal dilatation, and artery occlusion through reduction of adenosine and increase of TNAP with mineralization-related osteoclastogenesis in the affected arterial wall.

Of the 8 variants of $N T 5 E$ reported in CALJA patients, 5 are frameshift, splice junction or nonsense mutations, which may disrupt the production of intact protein. ${ }^{6-10}$ The disease is thought to be caused by the loss of enzyme 


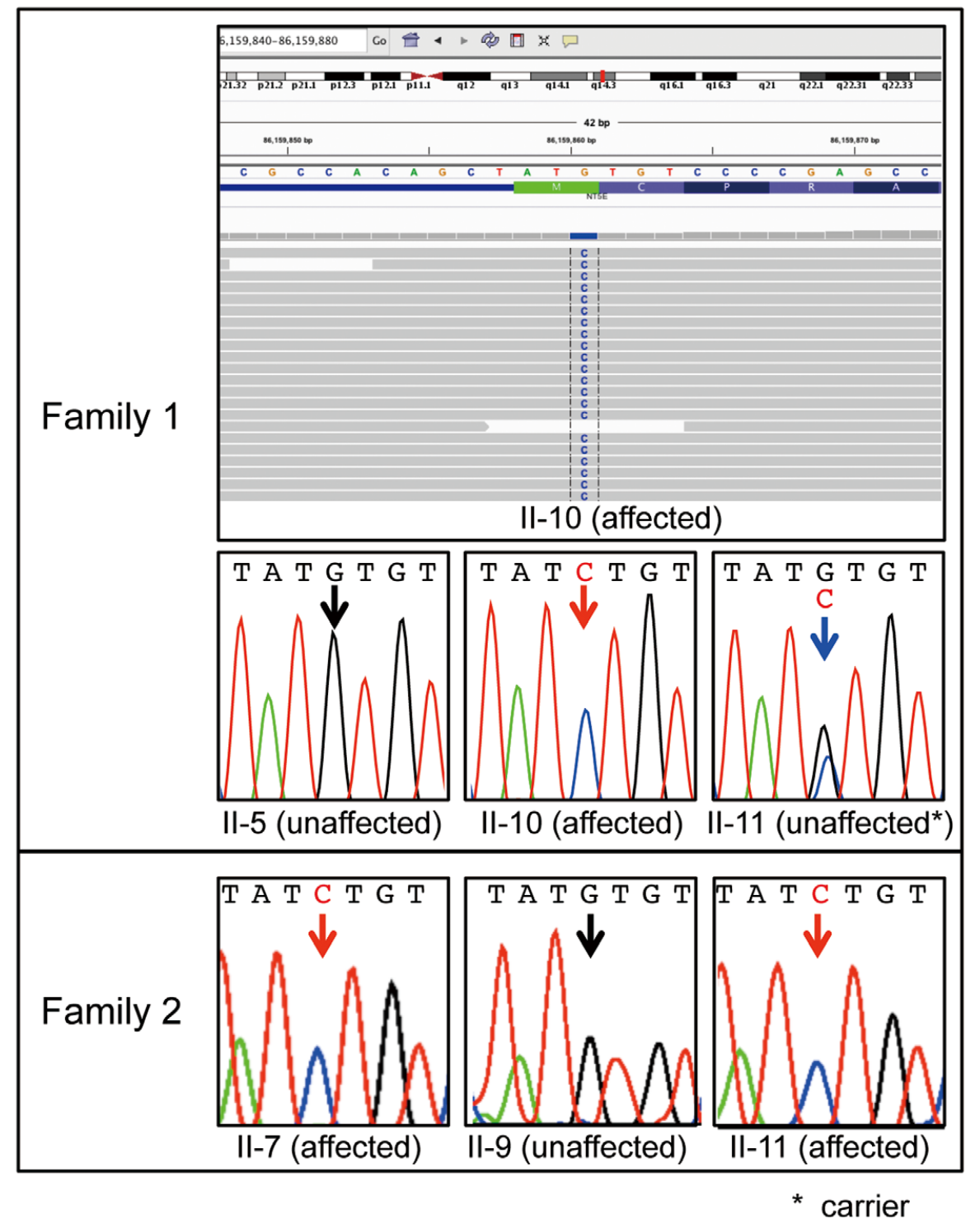

Figure 3. Identification and confirmation of the NT5E variant in Family 1 and Family 2. Data of II-5, II-10 and II-11 (listed in Figure 1A pedigree) in Family 1 and II-7, II-9, I-11(listed in Figure 2A pedigree) in Family 2 are shown. The Integrative Genome Viewer image shows sequencing reads that support the pathogenic variant identified in Patient II-10 in Family 1 (Upper Panel of Family 1). The identified C.3G>C displays homozygous and maps within NT5E exon 1. The mean coverage of the region is 63. Electropherograms show the NT5E genotypes in both families (Lower Panel of Family 1 and Family 2). Red and blue arrows indicate homozygous and heterozygous pathogenic variants, respectively. Black arrows indicate wild-type sequences.

activity, which is consistent with genetically recessive inheritance. The variant c. $3 \mathrm{G}>\mathrm{C}$, found in the current 2 families, is in the translation start site of NT5E. This type of variant disrupts the initiation codon and may lead to the use of alternative start site upstream or downstream of the transcript, or to be loss of translation. ${ }^{16}$ In the transcript, there is no ATG codon in the $5^{\prime}$-UTR, but is at 172-bp downstream of the original start site, which could be used as an alternative start codon. However, its product might be a truncated CD73 protein with partial lack of the nucleotidase domain. Thus, we speculate that the variant causing CALJA is a loss-of-function mutation.

The symptoms in the lower extremities of the affected members of these families were more severe, especially in the patient with CLTI in Family 1, than is usually seen in other patients. However, the genotype-phenotype relationship is still unknown because only a few cases have been reported. ${ }^{6-10}$ Because the disease is late-onset, other factors might also contribute to the severity and timing of onset. Based on the allele frequency, there might be undiagnosed patients with the homozygous variant of NT5E. In order to elucidate the pathophysiology of this disease and vascular calcification (medial calcification) and to develop treatment and prevention, the awareness of CALJA to vascular specialists and accumulation of such patients are important. 


\section{Conclusions}

In the present study, we identified members of 2 families in Japan with the same NT5E mutation who were diagnosed with CALJA. The genetic mutation pattern we found was unique compared with NT5E mutations reported in other countries. These CALJA patients also had relatively severe disease in terms of ischemic symptoms of the lower limbs, and 3 patients needed tibial artery bypass. Based on our estimation, many patients who suffer from PAD may be undiagnosed with CALJA, so vascular specialists need to recognize CALJA as a cause of lower limb ischemia, including CLTI.

\section{Disclosures}

N.A. is a member of Circulation Journal' Editorial Team.

\section{Conflict of Interest / Funding}

None.

\section{IRB Information}

Approved by the Ethics Committee of the Asahikawa Medical University (17091-2) followed by Yamagata University.

\section{References}

1. Magnus-Levy A. Ueber ungewöhnliche Verkalkung der Arterien (Arterienverkalkung ohne primäre Arteriosklerose?). Dtsch Med Wochenschr 1914; 40: 1305-1309 (in German).

2. Levitin J. A case of arterial and periarticular calcinosis of unknown etiology. Radiology 1945; 44: 489-494.

3. Sharp J. Heredo-familial vascular and articular calcification. Ann Rheum Dis 1954; 13: 15-27.

4. Nosaka H, Yamasaki G, Yamamoto K, Hayashi Y. A case of extensive calcification of the peripheral arteries. Yonago Acta Med 1974; 18: 191-197.

5. Mori H, Yamaguchi K, Fukushima H, Oribe Y, Kato N,
Wakamatsu T, et al. Extensive arterial calcification of unknown etiology in a 29-year-old male. Heart Vessels 1992; 7: 211-214.

6. St Hilaire C, Ziegler SG, Markello TC, Brusco A, Groden C, Gill $\mathrm{F}$, et al. NT5E mutations and arterial calcifications. $N$ Engl $J$ Med 2011; 364: 432-442.

7. Ichikawa N, Taniguchi A, Kaneko H, Kawamoto M, Sekita C, Nakajima A, et al. Arterial calcification due to deficiency of CD73 (ACDC) as one of rheumatic disease associated with periarticular calcification. J Clin Rheumatol 2015; 21: 216-220.

8. Zhang Z, He JW, Fu WZ, Zhang CQ, Zhang ZL. Calcification of joints and arteries: Second report with novel NT5E mutations and expansion of the phenotype. J Hum Genet 2015; 60: $561-$ 564.

9. de Nijs T, Albuisson J, Ockeloen CW, Legrand A, JeunE-mailtre $\mathrm{X}$, Schultze Kool LJ, et al. Isolated arterial calcifications of the lower extremities: A clue for NT5E mutation. Int J Cardiol 2016; 212: $248-250$

10. Yoshioka K, Kuroda S, Takahashi K, Sasano T, Furukawa T, Matsumura A. Calcification of joints and arteries with novel NT5E mutations with involvement of upper extremity arteries. Vasc Med 2017; 22: 541 - 543 .

11. Jin H, St. Hilarie C, Huang Y, Yang D, Dmitrieva NI, Negro A, et al. Increase activity of TNAP compensates for reduced adenosine production and promote ectopic calcification in the genetic disease ACDC. Sci Signal 2016; 9: Ra121.

12. Sasaki H, Yanagi K, Ugi S, Kobayashi K, Ohkubo K, Tajiri Y, et al. Definitive diagnosis of mandibular hypoplasia, deafness, progeroid features and lipodystrophy (MDPL) syndrome caused by a recurrent de novo mutation in the POLDI gene. Endocr $J$ 2018; 65: 227-238.

13. McNally EM. Genetics of vascular calcification. Circ Res 2011; 109: $248-249$.

14. Markello TC, Pak LK, St. Hilaire C, Dorward H, Ziegler SG, Chen MY, et al. Vascular pathology of medial arterial calcifications in NT5E deficiency: Implications for the role of adenosine in pseudoxanthoma elasticum. Mol Genet Metab 2011; 103: 44-50.

15. Zukowska P, Kutryb-Zajac B, Toczek M, Smolenski RT, Slominska EM. The role of ecto-5'-nucleotidase in endothelial dysfunction and vascular pathologies. Pharmacol Rep 2015; 67: 675-681.

16. Kochetov AV. Alternative translation start sites and hidden coding potential of eukaryotic mRNAs. BioEssay 2008; 30: 683691. 